\title{
A SIMULATION STUDY ON THE IMPACT OF PHYSICIAN STARTING TIME IN A PHYSICAL EXAMINATION SERVICE
}

\author{
Wheyming T. Song \\ Department of Industrial Engineering \\ National Ting Hua University \\ Hsinchu Taiwan 300, ROC
}

\author{
Aaron E. Bair
Department of Emergency Medicine
UC Davis Medical Center
Sacramento CA, USA

\author{
Mingchang Chih \\ Department of Industrial Engineering \\ National Ting Hua University \\ Hsinchu Taiwan 300, ROC
}

\begin{abstract}
The objective of our project was to improve the efficiency of a screening physical examination service of a large hospital system. We began with a detailed simulation model to explore the relationships between four performance measures and three decision factors. These included various dispatching rules, physician starting inquiry time, and scheduled patient arrival time. We then attempted to identify the optimal physician starting inquiry time. Our simulations show that (1) the three patient dispatching rules have negligible influence on any of the four outcome measures; (2) two types of patient arrival policies did not affect any of the four measures; (3) the proposed optimal physician starting inquiry time decreased patient wait time by $50 \%$ without increasing overall physician utilization. Based on these finding, we propose two improved policies. Policy 1 proposes changing the physician inquiry starting time from 10:30 am to 9:00 am. Policy 1 decreases patient wait time by $50 \%$ without increasing overall physician utilization and total physician shift time. Policy 2 suggests the postponement or removal of the physician inquiry stage but patients with abnormal results will be scheduled for further inspection.
\end{abstract}

\section{Introduction}

Physical examination (PE) services are commonly used for routine pre-employment and annual screening examinations for employees in various institutional settings throughout Taiwan. These services are time consuming for both the patients and the clinical personnel who work at the medical clinics. As such, maximal patient throughput and physician efficiency are of significant importance. Although the original motivation of this study was to improve the efficiency of a specific PE service, the insights that have been gained through this research is of more general value. The goal of this paper is to share these insights and to propose improved strategies which have wide applicability.
The PE service considered in this analysis consists of a routine medical physical examination which includes three ordered stages: (1) registration stage, (2) a series of diagnostic sub-stages (xray, ultrasound, blood analysis, and electrocardiogram) where the four sub-stages do not require any particular order, and finally (3) a physician inquiry stage. The three ordered stages are illustrated in Figure 1. We define "the confined constraint" to be the constraint that a PE service includes the three stages; that is, removing any stage from the PE service is not an option.

Original policies at the studied PE service are as follows: 20 (up to 25) patients are scheduled per morning (all patients are required to stop from eating or drinking after 9:00 pm the day before their clinical exam); all patients are requested to arrive at the hospital at 8:00 am. Moreover, the physician inquiry starting time (pist) at stage (3) for the PE service is $10: 30 \mathrm{am}$.

The goal of this study is to improve the efficiency of a PE service in terms of the following four measures: (a) physician utilization, $p_{u}$, which is defined as the probability that the doctor in the inquiry stage is busy (b) the mean of physician total shift time in the inquiry stage, $\mathrm{E}(D)$, where $D$ is the time that the doctor serves in the inquiry stage. (c) the mean of a randomly selected patient wait time in system, $\mathrm{E}(W)$, where $W=W_{i}$, with probability $1 / 20, i=1,2, \ldots, 20$. and (d) the patient prolonged wait rate, which is measured by the probability that a patient will stay in the clinic more than 150 minutes, $\mathrm{P}(W>150)$. We remind readers that the wait time should be defined carefully because wait time for all patients is not always in a steady state. We defined wait time to be the composition of all patient wait times in this paper. That is, we used notation " $W$ " as "a randomly selected patient wait time" rather than any particular patient's wait time because the $i^{\text {th }}$ patient wait times, $i=1,2, \ldots, 20$, are in a transient, rather than steady state.

We quantify the impact of these four measures with respect to three decision variables: (i) patient dispatching rules, (ii) physician inquiry starting time and (iii) scheduled patient arrival time. Different scenarios with respect to each 


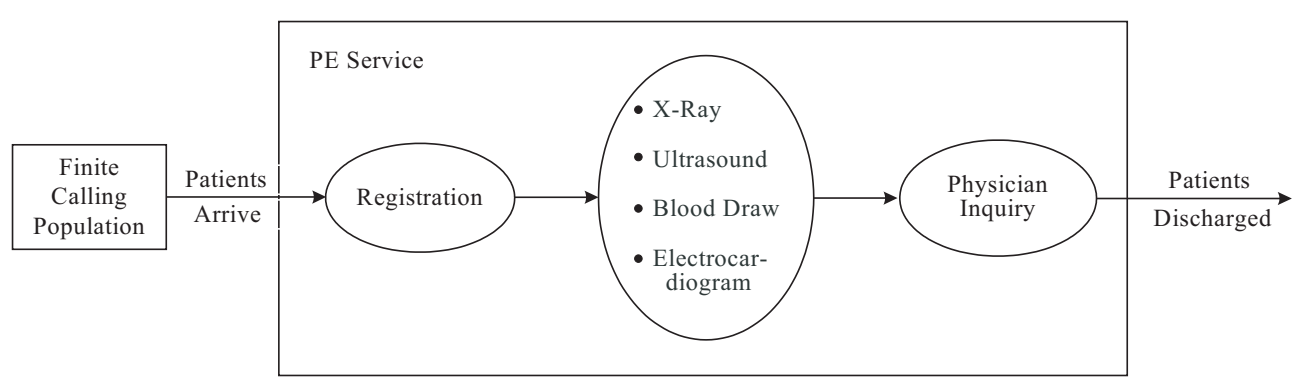

Figure 1: The PE Service Framework

policy are given below. We consider three scenarios for the patient dispatching rule for any patient to choose the next service in the diagnostic sub-stages: service in random order (Random) which is the original scenario, longest-processingtime-first (LPT), and shortest-processing-time-first (SPT). A total of 31 scenarios considered for the physician inquiry starting times are $t=0,5,10, \ldots, 150$; data are collected every 5 minutes from 8:00 to 10:30 am. Two scenarios of patient arrival policies are arrivals in one batch (which is the policy currently in place, referred to as "original") and arrivals in two batches (referred to as "staggered"). The original arrival policy requires all patients to arrive at the hospital at 8:00 am. The staggered arrival policy requires half of the patients to arrive at the hospital at 8:00 am and the other half of the patients to arrive at the hospital at 9:00 am.

Regarding methods, we first built a discrete-event simulation model to model the system illustrated in Figure 1. We then select the best physician inquiry starting time to reach multiple goals. Specifically, two types of goals are simultaneously considered in the objective function. From the physician/clinic point of view, we would like to maximize physician utilization and minimize physician total shift time; from the patients' point of view, we would like to minimize patient wait time and prolonged wait rate. The integrated measure that combining these four measures is adopted in the goal programming problem in the paper.

In our model we considered the various feasible, optimal, and ideal solutions. The feasible solution was the minimum requirement for clinical efficiency, whereas the optimal solution was obtained among all feasible solutions within confined constraints. The ideal solution, however, was obtained via relaxing existing confined constraints. Once a potential solution was obtained, we would then consider whether it was a compromise solution between two performance measures and how we could solve any conflicts in order to identify an ideal solution. We solved any identified conflicts by thinking beyond the existing confined constraints.
Figure 2 illustrates the performance measures, decision variables, methods, solution types adopted in this paper. The framework shown in Figure 2 could also be used as a generalized framework for any clinic service system.

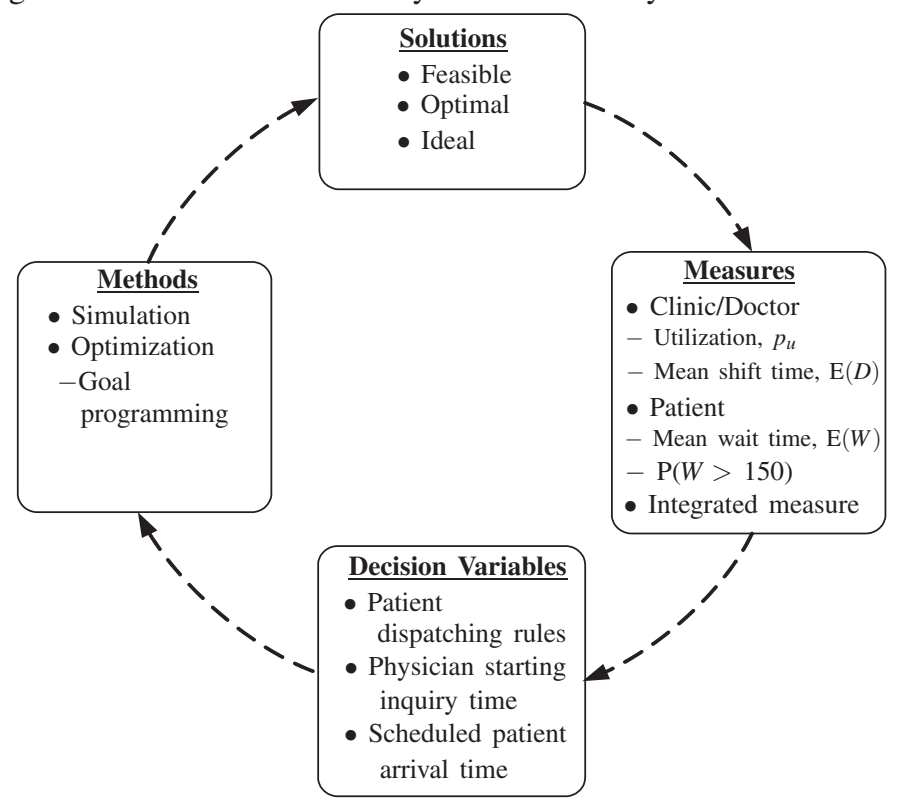

Figure 2: A framework of solutions, measures, decision variables, and methods for clinic services.

\section{Literature Review}

There have been many studies applying computer simulation in health care systems. For example, Kropp and Carlson(1977) discussed a recursive optimization-simulation approach of ambulatory health care settings. Wullink et al.(2007) developed a discrete-event simulation model to investigate the optimal policy for reserving operating room capacity. In the last few years, several articles have been devoted to the study of a comprehensive survey for ap- 
Song, Bair, and Chih

Table 1: The fitting probability distribution of random variables in the physical examination services

\begin{tabular}{c|cccc}
\hline & Random Variable & $\begin{array}{c}\text { Fitting Probability } \\
\text { Distribution }\end{array}$ & $\begin{array}{c}\text { Mean } \\
(\mathrm{min} .)\end{array}$ & $\begin{array}{c}\text { Standard Deviation } \\
(\mathrm{min} .)\end{array}$ \\
\hline Arriving Process & Patient Inter-Arrival Time & $0.01+28.13$ beta $(0.74,3.4)$ & 5.1 & 4.8 \\
Service Process & & & & \\
Stage 1 & Registration & $6.19+5.82$ beta( $(0.52,0.97)$ & 8.2 & 1.8 \\
& & & \\
Stage 2 & X-Ray & uniform $(5.43,8.74)$ & 7.1 & 1.0 \\
& Ultrasound & uniform $(3.53,11.08)$ & 7.3 & 2.2 \\
& Blood Draw & $1.34+4.87$ beta(0.38, 1.18) & 2.5 & 1.3 \\
& Electrocardiogram & $3.34+3.79$ beta( $(0.63,0.94)$ & 5.0 & 1.2 \\
Stage 3 & Doctor inquiry & $4.86+5.21$ beta(0.64, 0.78) & 7.2 & 1.7 \\
\hline
\end{tabular}

plying simulation in health care systems. England and Roberts(1978) provided a framework of computer simulation in health care. Jun et al.(1999) published a review of applications of simulation in health care, covering the early 1960 s to the late 1990s. Fone et al.(2003) conducted a systematic review to evaluate the extent, quality and value of computer simulation modeling in population health and health care delivery.

\section{Methods}

In this section we first discuss how we constructed our simulation model. Additionally, we discuss the selection of a best system according to multiple goals.

\subsection{Simulation Model}

To investigate the PE services, we first built a simulation model to estimate the four performances discussed above. We used SIGMA (see Schruben, 1983 and 1990) rather than other simulation software because it can be automatically translated into $\mathrm{C}$ code, and thus allowing our simulation models to run quickly and efficiently.

\subsubsection{Input Modeling}

SSimulation modeling first requires the collection of actual data. For each patient, we recorded the registration time, and the time the patient began and ended each stage of their examinations. These data were used to fit distributions for the patient inter-arrival times; processing times for the registration, x-ray, ultrasound, blood draw, electrocardiogram, and physician inquiry.

The hypothesized probability density functions considered in this paper are functions of uniform or beta distributions because these two types of distributions have a finite range. Chi-square tests for goodness-of-fit (Montgomery and Runger, 2006, p-316), a commonly used measurement of how well the sample data fit a hypothesized probability density function, were used to evaluate the fits of various candidates probability distributions to the observed data. The Chi-square statistic is $\sum_{i=1}^{k}\left(e_{i}-o_{i}\right)^{2} / e_{i}$, where $o_{i}$ is the observed value, $e_{i}$ is the theoretical value, and $k$ is the number of intervals. A low Chi-square value indicates a good fit. The selected distributions on the collected data are summarized in Table 1. The maximum-likelihood-estimation are used to estimate the distribution parameters.

There are two types of random processes involved in the studied PE service: arrival processes and service processes. In the arrival process, we fit the patient interarrival times as $0.01+28.13$ beta $(0.74,3.4)$ with a mean time of 5.05 minutes. In the service processes, the fitted distribution for the registration, x-ray, ultrasound, blood draw, electrocardiogram, and physician inquiry are listed in Table 1. The corresponding mean and variances for each fitted distribution are also shown.

\subsubsection{Different Scenarios}

The purpose of the simulation experiments implemented in this analysis is to evaluate the system performance under three clinic policies with different scenarios.

As mentioned earlier, three types of clinic policies are: (i) three patient dispatching rules; (ii) 31 physician inquiry starting times $t=0,5,10,15,20, \ldots, 150$, where $t=0$ denotes physician inquiry starting time being 8:00 am; (iii) two types of patient arrival policies. Therefore, there are $3 \times 31 \times 2=186$ combinations of scenarios for simulation experiments. We executed 5000 replications of the simulation experiments for each scenario. One replication generates one estimator of performance measure. Based on 5000 replications, we can obtain the estimates and the standard error of each performance measure, such as the patient mean wait time. To minimize sampling error, Common Random Numbers (CRN, which is a variance reduction technique, see Law, 2007 and Shechter et al., 2006) are used to in- 


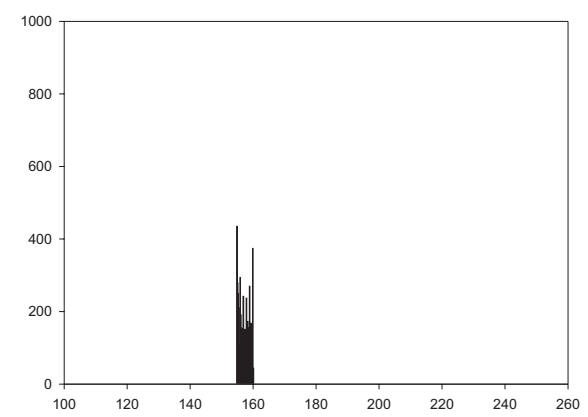

(a) the 1st patient: mean $=157.3$, s.d. $=1.7$

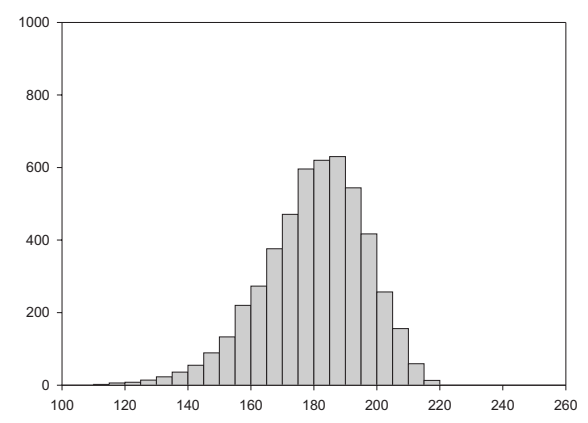

(c) the 11th patient: mean=180.1, s.d. $=16.4$

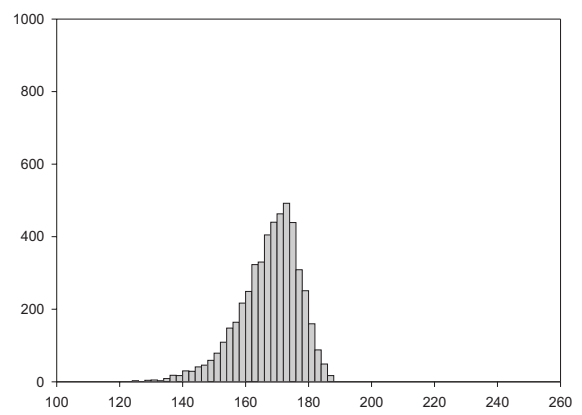

(b) the 5th patient: mean $=167.4$, s.d. $=9.6$

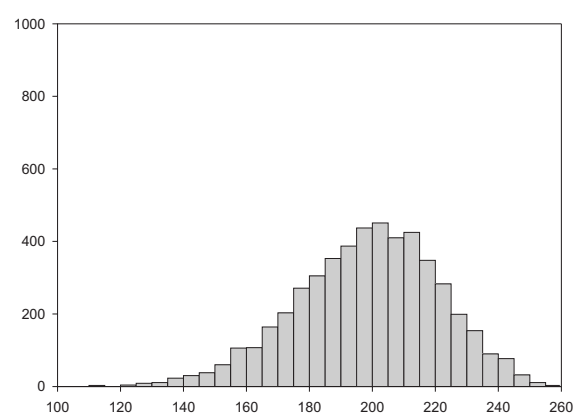

(d) the 20th patient: mean $=198.3$, s.d. $=23$

Figure 3: The transient behavior of patient waiting times (minutes) via the histograms of the 1st, 5th, 11th and 20th patients under original policy. $\mathrm{x}$-axis: waiting time in minutes. $\mathrm{y}$-axis: frequency.

vestigate the performance under different scenarios in these simulation experiments. To carry out CRN, we have to use the same random stream set $\overline{\mathbf{R}}$ for all 186 combinations of scenarios in a replication. There are eight random streams in a set, i.e. $\overline{\mathbf{R}}=\left\{\mathbf{R}_{1}, \mathbf{R}_{2}, \ldots, \mathbf{R}_{8}\right\}$. Streams $\mathbf{R}_{1}, \mathbf{R}_{2}, \ldots, \mathbf{R}_{7}$ are used for generating the seven distributions, as shown in Column 3 in Table 1; and stream $\mathbf{R}_{8}$ is used for generating each registered patient's next sub-stage in Stage 2.

\subsection{Stochastic Optimization}

This section discusses the selection of the best physician inequity time via solving a goal programming problem, where the objective function needed to be estimated. The multiple goals we considered simultaneously are the maximization of the physician utilization; the minimization of the patient wait time, the prolonged wait rate, and the physician total shift time.
A prototype multiple goals programming problem is defined as (P1) (Hillier and Lieberman, 2001, p-332):

$$
\begin{aligned}
& \text { (P1) Min: } \quad c_{1}\left[c_{2} y_{1}^{-}+y_{2}^{+}\right]+\left[c_{2} y_{3}^{+}+y_{4}^{+}\right] \\
& \text {subject to : } \\
& p_{u}-\left(y_{1}^{+}-y_{1}^{-}\right)=g_{1} \\
& \mathrm{E}(D)-\left(y_{2}^{+}-y_{2}^{-}\right)=g_{2}, \\
& \mathrm{E}(W)-\left(y_{3}^{+}-y_{3}^{-}\right)=g_{3} \\
& \mathrm{P}(W>150)-\left(y_{4}^{+}-y_{4}^{-}\right)=g_{4}, \\
& y_{i}^{+}, y_{i}^{-} \geq 0, i=1,2,3,4 \\
& t=0,5, \ldots, 150
\end{aligned}
$$

where the four performance measures $p_{u}, \mathrm{E}(D), \mathrm{E}(W)$, and $\mathrm{P}(W>150)$ are functions of $t ; c_{1}$ and $c_{2}$ are pre-specified values; $g_{1}$ is the upper bound of utilization; $g_{2}, g_{3}, g_{3}$ are lower bounds of $\mathrm{E}(D) \mathrm{E}(W)$, and $\mathrm{P}(W>150)$, respectively.

The values $c_{1}, c_{2}$ are used as the penalty costs if the response values do not satisfy the goal. We set $c_{1}=2$ to indicate that one unit of physician benefit (such as utilization) is as important as 2 units of patient benefit (such as prolonged 


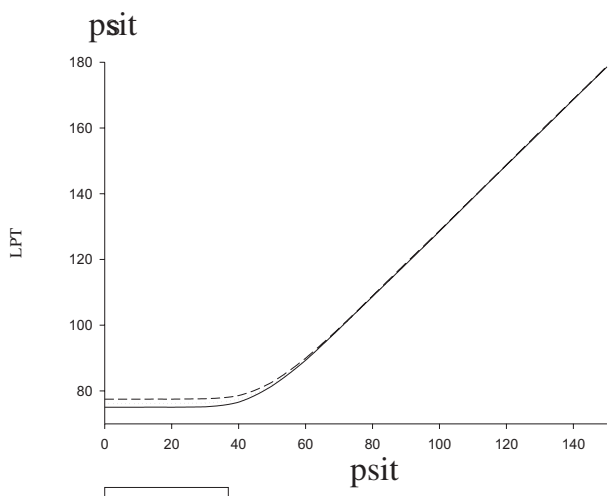

(a) $p_{u}$ vs. psit

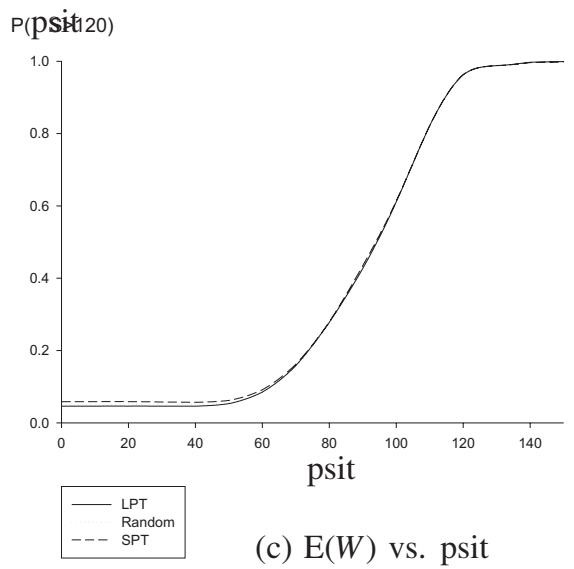

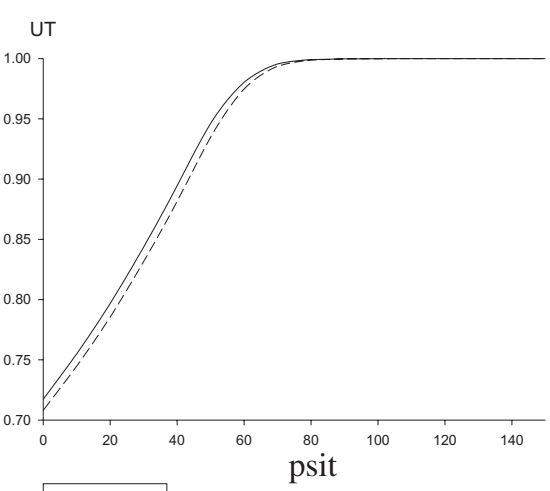

(b) $\mathrm{E}(D)$ vs. psit

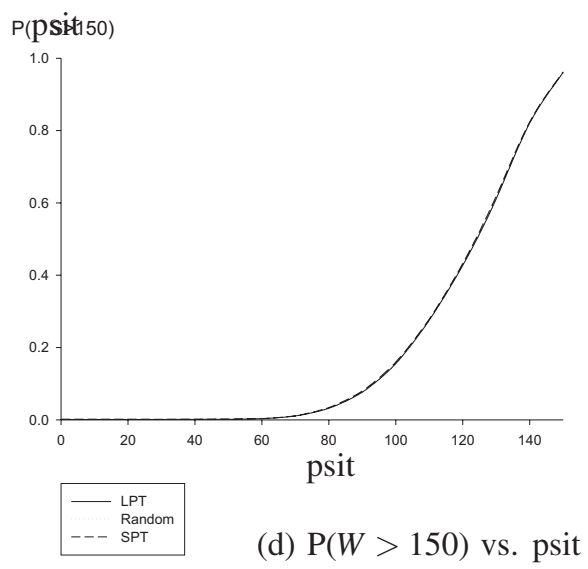

Figure 4: Four performance measures as functions of physician starting inquiry time (psit). $p_{u}$ : mean physician utilization; $\mathrm{E}(D)$ : mean total service time for the physician inquiry; $\mathrm{E}(\mathrm{W})$ : patient mean waiting time; $\mathrm{P}(\mathrm{W}>150)$ : patient prolonged wait-time rate

wait rate). We set $c_{2}=5$ to indicate that one unit (one percent) of physician utilization is as important as 5 units (minutes) of total shift time, and similarly, one unit (percent) of patient prolonged wait rate is rated as important as 5 units (minutes) of mean wait time. We set the upper bound of utilization as $g_{1}=1$. We set $g_{2}=g_{3}=g_{4}=0$ because the true lower bounds for the mean patient wait time and the mean physician total shift time wait time are unknown, and setting the values $g_{2}, g_{3}, g_{4}$ lower than their true lower bounds will not change the solution of problem $(\mathrm{P} 1)$.

Inserting $c_{1}=2, c_{2}=5, g_{1}=1, g_{2}=g_{3}=g_{4}=0$ in the problem, and unifying the objective function and constraints of (P1), we can rewrite problem (P1) as (P2):

$$
\begin{gathered}
\text { Min } z(t)=2\left[5\left(1-p_{u}\right)+\mathrm{E}(D)\right]+5 \mathrm{E}(W)+\mathrm{P}(W>150) \\
\text { subject to }: t=0,5, \ldots, 150
\end{gathered}
$$

where we call $z(t)$ the integrated performance measure that includes multiple goals. It is noted that $z(t)$ needed to be estimated via simulation.

We can solve problem (P3) via stochastic optimization. According to Goldsman and al. (1991), three types of methods for selecting the best systems via stochastic optimization are interactive approach (IA), ranking and selection (R\&A), and multiple comparison procedures (MCPs). The concept of standard error underlines all three approaches, but the differ in that IA, R\&A, and MCPs are based on estimation, optimization, and inference, respectively. In this paper, we 


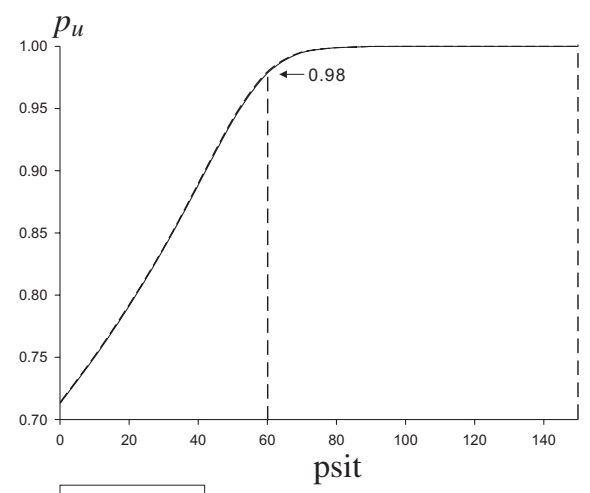

(a) $p_{u}$ vs. psit

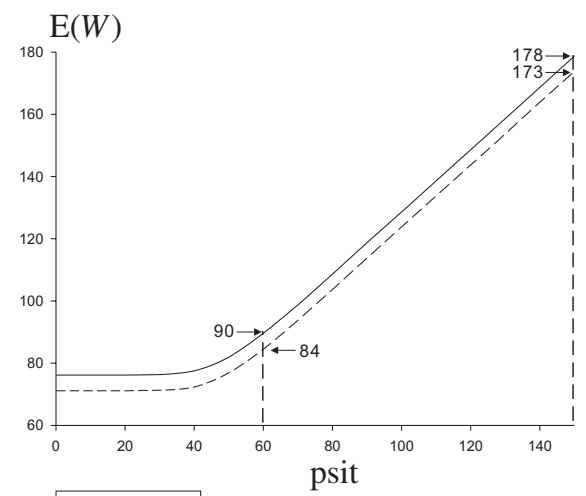

(c) $\mathrm{E}(W)$ vs. psit

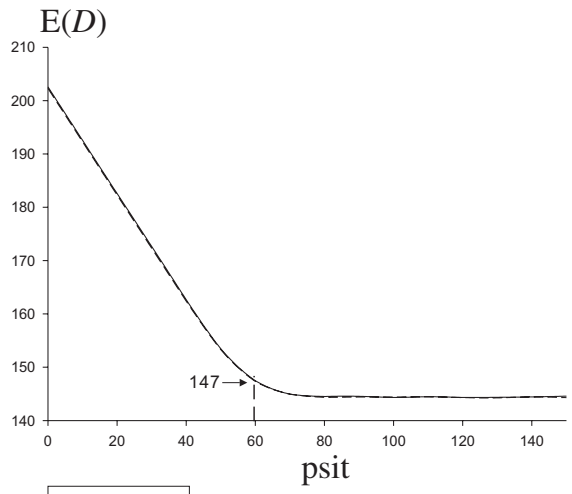

(b) $\mathrm{E}(D)$ vs. psit

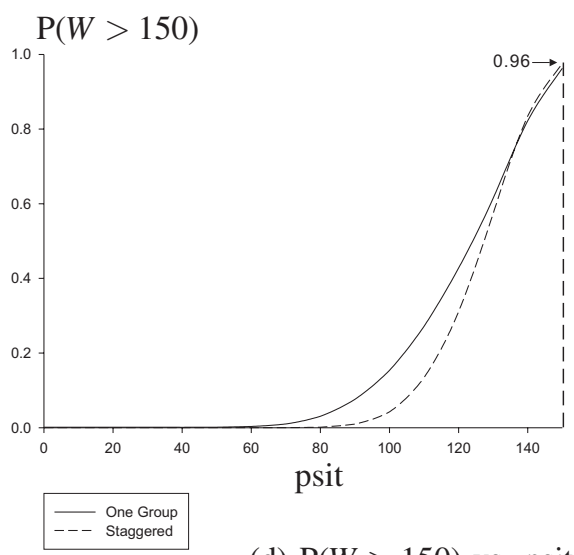

Figure 5: Four performance measures between the original and staggered registration policies

adopt the IA approach to select the optimal physician inquiry time. That is, we first simulate 31 scenarios with respect to 31 values of $t$, and then select the best $t$ in terms of the integrated performance measure.

\section{Simulation Results}

In this section, we demonstrate the simulation results including the transient behavior of patient wait time and the impact of three decision variables on the four performances. The simulation results demonstrate that the original PE service is not a stationary system. Among the three decision variables, only physician inquiry starting time has significant impact on the four performance measures.

\subsection{Transient Behavior of Patient Waiting Time}

The transient behavior of $i^{\text {th }}, i=1,5,11,20$ patient wait time in the original PE service is illustrated in Figure 3, in which the estimated mean and variance are stated below each of the four plots. As expected, the distributions of the $i^{\text {th }}, i=1,5,11,20$ patient wait time in the PE service are not identical. Further, both the means and variance of the patient wait time in the PE services do not converge to a fixed value. Specifically, the mean and variance for the first patient are 157.27 and 2.76 minutes (see Figure 3 (a)); and for the last patient are 198.3 and 529 minutes (see Figure 3 (d)), respectively. That is, patient wait times are in transient state, and not in steady state.

\subsection{Impact of Dispatching Rules}

We investigate whether different dispatching rules affect patients and clinic performance. Recall that the original patient dispatching rule is random; patients randomly choose the next stage for service. Alternative dispatching rules that we considered were LPT and SPT. 


\section{Song, Bair, and Chih}

The results via the four plots in Figure 4 show that the three patient dispatching rules have negligible influence on physician utilization, physician total shift time in the inquiry stage, patient wait time, and the patient prolonged wait rate. The $\mathrm{x}$-axis of the four plots in Figure 4 is physician inquiry starting time (pist) and the y-axis for plots (a), (b), (c), (d) are $p_{u}, \mathrm{E}(D), \mathrm{E}(W)$ and $\mathrm{P}(W>150)$, respectively. If pist is 9:00 am (corresponding to 60 in the $\mathrm{x}$-axis), then the LPT policy provides a shorter $\mathrm{E}(W)$ than both Random and SPT policies with respect to both $\mathrm{E}(W)$ and $p_{u}$ performances. Although this difference is statistically significant, there is no practical difference.

\subsection{Impact of Staggered Arrival Policy}

We investigate whether two types of patient arrival policies: (1) original (one group) policy and (2) staggered policy affect four measures via Figure 5, where one group policy represents that all patients' registration time is 8:00 am and the staggered policy adopts two registration times, 8:00 am and 9:00 am. Figure 5 is similar to Figure 4 with the same $\mathrm{x}$-axis and $\mathrm{y}$-axis, except that two plots in Figure 5 are for two types of patient arrival schedules.

The two plots in Figures 5 (a) and (b) are almost identical; two types of patient arrival policies did not affect any of the four measures. Although two plots in Figures 5 (c) and (d) are not identical, their differences are not practically significant.

\subsection{Impact of Physician Starting Inquiry Time}

We investigate whether physician inquiry starting time affect patients and clinic performance. Plots in Figures 4 and 5 clearly demonstrate that the physician inquiry starting time has a strong impact on performance. For example, the mean physician utilization $p_{u}$ rises from 0.70 to 0.98 to 1.0 as the pist increases from 8:00 am to 9:00 am to 9:10 am, as seen in Figure 5(a). The mean physician total shift time decreases from 200 minutes to 147 minutes as the pist increases from 8:00 am to 9:00 am, seen in Figure 5(b). The mean patient wait time increases from 90 minutes to 178 minutes as the pist increases from 9:00 am to 10:30 am. The patient prolonged wait rate increases from 0 to 0.96 as pist increases from 9:00 am to 10:30 am.

\subsection{Meta-models}

Due to the fact that the physician inquiry starting time has a strong impact on four performance measures, we constructed regression models for the four measures as functions of physician inquiry starting time. Such regression models are also referred to as meta-models (Friedman and Pressman, 1988 and Kleijnen, 2007) because they are models based on simulation models. The advantage of a meta-model is its functional form which can be used to approximate performance value as a function of any physician inquiry starting time. We did not intend to replace the unknown performance measures by the corresponding meta-models into the goal programming problem (P2) and solve it for optimal solution because stochastic optimization approach (as we discussed earlier) is more appropriate, in general.

For each performance measure, we consider two types of meta-models: quadratic and mixed (linear and quadratic). That is, the physician utilization, $p_{u}$; the mean physician total shift time, $\mathrm{E}(D)$; the mean patient wait time, $\mathrm{E}(W)$; and the patient prolonged wait rate, $\mathrm{P}(W>150)$ are written as functions of $t$, physician inquiry starting time (pist) in two types of functional forms. The value of $R^{2}$ inside the parenthesis is a commonly used quality measure to indicate the fitness of the corresponding meta-model.

1. Fitted Physician Utilization, $\hat{p}_{u}$ :

(a) Quadratic $\left(R^{2}=0.98\right)$ :

$$
\hat{p}_{u}=0.704+0.00566 t-0.000026 t^{2}
$$

(b) $\quad$ Mixed $\left(R^{2}=0.99\right)$ :

$$
\hat{p}_{u}= \begin{cases}0.711+0.0043 t, & 0 \leq t \leq 60 \\ 0.9993, & 60<t \leq 150\end{cases}
$$

2. Fitted Physician total shift time, $\hat{\mathrm{E}}(D)$ :

(a) Quadratic $\left(R^{2}=0.98\right)$ :

$$
\hat{\mathrm{E}}(D)=201-1.13 t+0.00524 t^{2}
$$

(b) $\quad$ Mixed $\left(R^{2}=0.99\right)$ :

$$
\hat{\mathrm{E}}(D)= \begin{cases}203-1.11 t+0.00284 t^{2}, & 0 \leq t \leq 60 \\ 144.5, & 60<t \leq 150\end{cases}
$$

3. Fitted Waiting Time in System, $\hat{\mathrm{E}}(W)$ :

(a) Quadratic $\left(R^{2}=0.99\right)$ :

$$
\hat{\mathrm{E}}(W)=72.4+0.0777 t+0.00444 t^{2}
$$

(b) $\quad$ Mixed $\left(R^{2}=0.99\right)$ :

$$
\hat{\mathrm{E}}(W)= \begin{cases}76.5, & 0 \leq t \leq 40 \\ 45.4+0.68 t+0.00144 t^{2}, & 40<t \leq 150\end{cases}
$$

4. The fitted probability of the patient prolonged wait time, $\hat{\mathrm{P}}(\mathrm{W}>150)$ :

(a) Quadratic $\left(R^{2}=0.99\right)$ :

$$
\hat{\mathrm{P}}(W>150)=0.0862-0.0072 t+0.000086 t^{2}
$$

(b) $\quad$ Mixed $\left(R^{2}=0.99\right)$ :

$$
\begin{aligned}
& \hat{\mathrm{P}}(W>150) \\
= & \begin{cases}0.00265, & 0 \leq t \leq 60 \\
0.522-0.0169 t+0.000134 t^{2}, & 60<t \leq 150\end{cases}
\end{aligned}
$$



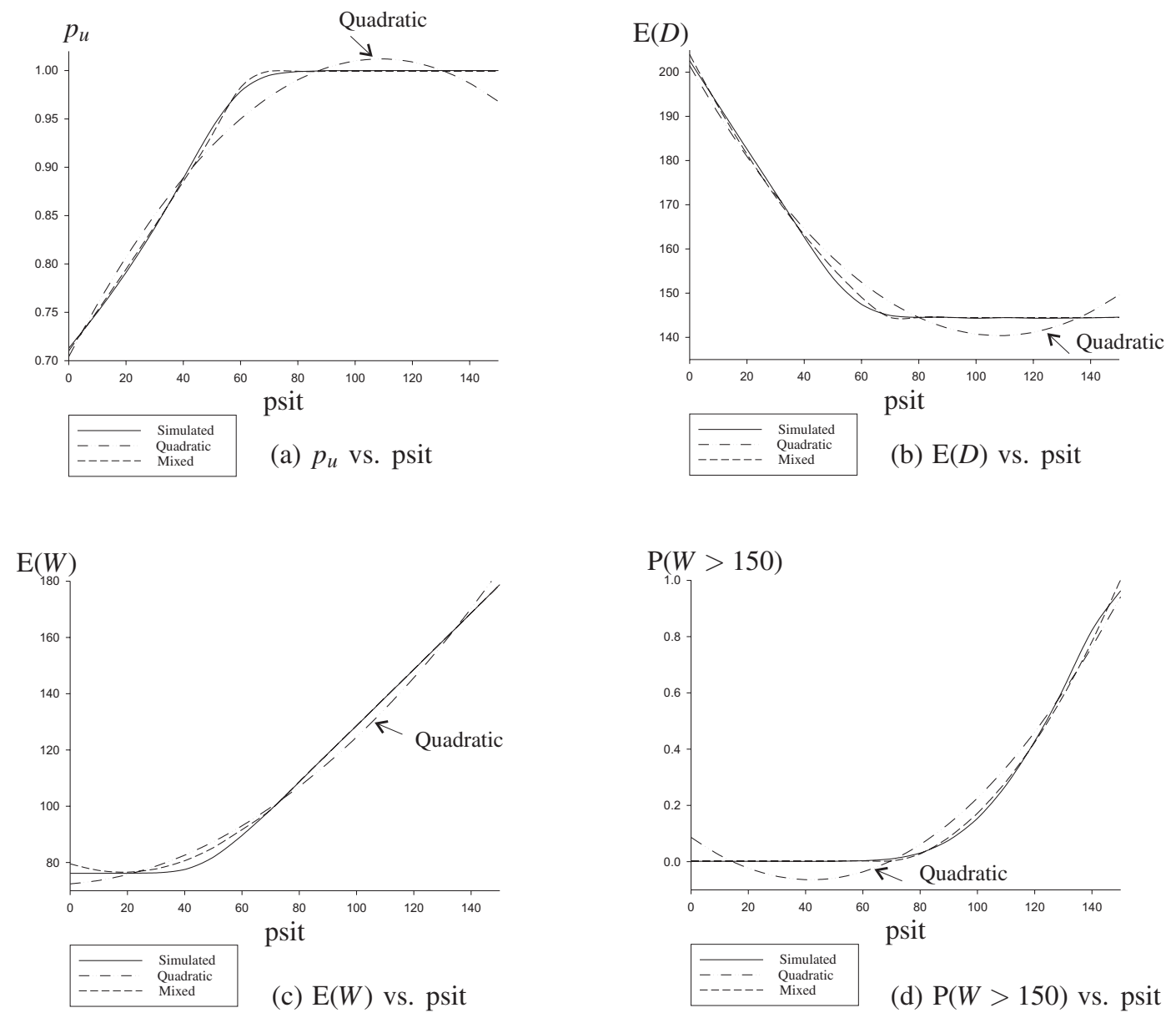

Figure 6: Simulated and two meta-models. (Note: Quadratic models provide some infeasible values.)

We discuss the values of $R^{2}$ for the two types of metamodels. The $R^{2}$ for four quadratic models are above 0.98 . Alternatively, the $R^{2}$ for the four mixed meta-model are also above 0.98 . Although both types of meta-models essentially have the same $R^{2}$, mixed models fit better than quadratic models (see Figure 6). Please note that $R^{2}$ cannot measure the appropriateness of the models. For example, in Figure 6 (a), we observe that the quadratic model suggests some value of $p_{u}$ larger than the feasible upper bound 1 , and in Figure 6 (d), the quadratic model suggests some value of $\mathrm{P}(W>150)$ lower than the feasible lower bound 0 . That is, quadratic models suggest some infeasible values for $p_{u}$ and $\mathrm{P}(W>150)$, although $R^{2}$ is as high as 0.98 .

\section{Proposed Policies}

In this section, we provide two policies to improve the PE services. Policy 1 provides an optimal physician inquiry starting time under the confined constraint and policy 2 is an innovative approach by relaxing the confined constraint.

\subsection{Policy 1: Determining the Optimal Physician Starting Inquiry Time}

In this section, we discuss the determination of the optimal physician staring inquiry time under the confined constraint in which the three stages are included in the PE services. The improved policy is the optimal solution for the goal programming (P1) using the IA approach. We especially estimate the objective function (shown in problem P1) with respect to 31 possible physician inquiry starting times (every 5 minutes between 8:00 am to 10:30 am).

The comparison between the original policy and Policy 1 is given in Table 2. The two policies differ in that the physician inquiry starting time is 10:30 vs. 9:00 am; both policies apply random dispatching rules and no staggered 
Song, Bair, and Chih

Table 2: Comparison between the original and two improved policies

\begin{tabular}{|c|c|c|c|c|c|c|c|}
\hline & \multicolumn{3}{|c|}{ Policy } & \multicolumn{4}{|c|}{ Performance } \\
\hline & $\begin{array}{l}\text { Dispatching } \\
\text { rule }\end{array}$ & $\begin{array}{l}\text { Staggered } \\
\text { arrival? }\end{array}$ & $\begin{array}{c}\text { Physician starting } \\
\text { inquiry time }\end{array}$ & $\begin{array}{l}\text { mean } \\
\text { Physician } \\
\text { utilization }\end{array}$ & $\begin{array}{l}\text { mean } \\
\text { Physician total } \\
\text { shift time }\end{array}$ & $\begin{array}{c}\text { mean } \\
\text { Patient } \\
\text { wait time }\end{array}$ & $\mathrm{P}(\mathrm{W}>150)$ \\
\hline Original & Random & no & $10: 30$ & 1 & 145 & 179 & 0.96 \\
\hline Policy 1 & Random & no & 9:00 & 0.98 & 148 & 90 & 0.003 \\
\hline Policy 2 & Random & no & not an issue & not an issue & not an issue & 65 & 0 \\
\hline
\end{tabular}

arrival rule. The optimal pist reduces the mean patient wait time by half (179 minutes to 90 minutes) and decreases the patient prolonged wait rate from 0.96 to 0.003 , but the physician utilization ( 1 vs. 0.98) and the mean physician total service time ( 145 vs. 148 minutes) essentially remain the same.

\subsection{Policy 2: An Innovative Approach}

At the end of the previous section, we derived policies in order to improve the efficiency of the PE service under the confined constraint. However, we believe that we have identified a further improvement to Policy 1. In this section, we explore an innovative approach that changes the original PE system by either postponing (after all diagnostic studies have been completed) or entirely deleting the physician inquiry stage (if there are no abnormalities to discuss) from the current PE service.

In the original PE service, decreasing the mean patient wait time and increasing physician utilization is a trade-off. Therefore, the optimal solution proposed was a compromise instead of an ideal solution for both patients and physicians. Recognizing this potential conflict and realizing that most of the laboratory and radiology results for each patient were not available in the inquiry stage, the proposed innovative idea was to remove or postpone the physician inquiry stage from the current PE service. After the individual patient receives his/her complete PE results via postal mail (normally one week after the patient receives the PE services), he/she would then be informed to return to the clinic for physician inquiry service only if abnormal results are identified.

From the patient point of view, the mean patient wait time will be decreased by more than half (from 179 minutes to 65 minutes, see the last row of Table 2). Further, the patient will receive more complete advice from the clinic as subsequent referral and scheduling of a return visit (if necessary) will be directed to the appropriate specialist. Thus, the return visits will be more time efficient for the patients.

\section{Conclusion}

Motivated by efficiency improvement of the physician inquiry stage, which is the bottleneck stage in a physical examination service, we investigated and modeled the system via a simulation model and quantified the impact of three types of decision policies in terms of performance measures. The simulation results show that the optimal physician inquiry starting time (pist) is 9:00 am, whereas the original pist is 10:30 am. The proposed pist reduces the mean patient wait time from the original 3 hours to 1.5 hours and decreases the prolonged wait rate (probability that patients wait more than 2.5 hours) from 0.96 to 0.003 . Nonetheless, the physician utilization and the mean total physician service time essentially remain the same.

Additionally, we recognized the conflict between physician utilization and patient wait time. We explored an innovative approach to remove or postpone physician inquiry stage from the original PE service. This resulted in an improved solution over what was found to be the "optimal" solution under the original system. Specifically, this meant that patient wait time decrease from 1.5 hour to 36 minutes without the requirement of additional clinic resources. Although the case studied in this paper is for a specific hospital, the advantage of applying an innovative approach, such as that proposed in this paper, is not limited to this hospital; that is, all similar PE services would benefit from the innovative approach suggested in this paper.

\section{REFERENCES}

England, W. and S. D. Roberts. 1978. Applications of computer simulation in health care. In Proceedings of the 1978 Winter Simulation Conference, eds. N. R. Nielsen, L. G. Hull, and H. J. Highland, 665-677. Institute of Electrical and Electronics Engineers.

Fone, D., S. Hollinghurst, M. Temple, A. Round, N. Lester, A. Weightman, K. Roberts, E. Coyle, G. Bevan and S. Palmer. 2003. Systematic review of the use and value of computer simulation model- 
ing in population health and health care delivery. Journal of Public Health Medicine, 25, 325-335.

Friedman, L.W. and I. Pressman. 1988. The metamodel in simulation analysis: can it be trusted? Journal of the Operational Research Society, 39, 939-948.

Goldsman, D., B. L. Nelson and B. Schmeiser. 1991. Methods for selecting the best system. In Proceedings of the 1991 Winter Simulation Conference, eds. B. L. Nelson, W. D. Kelton, and G. M. Clark, 177-186. Institute of Electrical and Electronics Engineers.

Hillier, F. S. and G. J. Lieberman. 2001. Introduction to operations research. New York: McGraw-Hill, Seventh Edition.

Jun J. B., S. H. Jacobsen, and J. R. Swisher. 1999. Application of discrete-event simulation in health care clinics: a survey. Journal of the Operational Research Society, 50, 109-123.

Kleijnen, J.P.C. 2007. DASE: Design and Analysis of Simulation Experiments. New York, New York: Springer, First Edition.

Kropp, D. H. and R. C. Carlson. 1977. Recursive modeling of ambulatory health care settings. Journal of Medical Systems, 1, 123-135.

Law, A.M. 2007. Simulation modeling and analysis. Boston, Massachusetts: McGraw-Hill, Fourth Edition.

Montgomery, D. C. and G. C. Runger. 2006. Applied Statistics and Probability for Engineering. New York: John Wiley \& Sons, Inc., Third Edition.

Schruben, L. W. 1983. Simulation modeling with event graphs. Communications of the Association of Computing Machinery, 26, 957-963.

Schruben, L. W. 1990. Simulation graphical modeling and analysis (SIGMA) tutorial. In Proceedings of the 1990 Winter Simulation Conference, eds. O. Balci, R. P. Sadowski, and R. E. Nance, 158-161. Institute of Electrical and Electronics Engineers.

Shechter, S. M., A. J. Schaefer, R. S. Braithwaite, and M.S. Roberts. 2006. Increasing the efficient of monte carlo cohort simulation with variance reduction techniques. Medical Decision Making, 26, 550-553.

Wullink, G., M. V. Houdenhoven, E. W. Hans, J. M. Oostrum, M. Lans, and G. Kazemier. 2007. Closing emergency operating rooms improves efficiency. Journal of Medical Systems, 31, 543-546.

\section{AUTHOR BIOGRAPHIES}

WHEYMING T. SONG is a professor in the Department of Industrial Engineering at the National Tsing Hua University in Taiwan. She received her undergraduate degree in statistics and masters degree in industrial engineering at Cheng-Kung University in Taiwan in 1979. She then received masters degrees in applied mathematics in 1983 and industrial engineering in 1984, both from the University of Pittsburgh. Dr. Song received her Ph.D. from the School of Industrial Engineering at Purdue University in 1989. She Joined Tsing Hua in 1990 after spending one year as a visiting assistant professor at Purdue IE. Her research interests are applied operations research; probability and statistics; and the statistical aspects of stochastic simulation, including input modeling, output analysis, variance reduction, and ranking and selection. <wheyming@ie.nthu.edu.tw>.

AARON E. BAIR is Associate Professor in the Department of Emergency Medicine at the University of California, Davis. His primary teaching and research interests focus on procedural competency in emergency airway management and emergency ultrasound. His current research is directed at workflow efficiency using computer modeling. <aebair@ucdavis.edu>.

MINGCHANG CHIH received his undergraduate degree in Industrial and Systems Engineering from Chung Yuan Christian University in Taiwan in June 2001. He then received Master's degree in Industrial Management from National Taiwan University of Science and Technology in Taiwan in June 2003. He is now a Ph.D. candidate at National Tsing Hua University. <d927805@oz .nthu . edu.tw>. 